\title{
Some properties and mappings on weakly $v$-Lindelöf generalized topological spaces
}

\author{
Mariam Abuage $^{a}$, A. Kiliçman ${ }^{b, *}$ \\ ${ }^{a}$ Institute for Mathematical Research, University Putra Malaysia, 43400 UPM, Serdang, Selangor, Malaysia. \\ ${ }^{b}$ Department of Mathematics, University Putra Malaysia, 43400 UPM, Serdang, Selangor, Malaysia. \\ Communicated by A. Atangana
}

\begin{abstract}
Our work aims to study weakly $v$-Lindelöf (briefly $w v$-Lindelöf) space in generalized topological spaces. Some characterizations of $w v$-Lindelöf subspaces and subsets are showed. Furthermore, we shall show that the $w v$-Lindelöf generalized topological space is not a hereditary property. Finally, the effect of some mappings and decompositions of continuity are studied. The main result that we obtained on is the effect of almost $(\nu, \mu)$-continuous function on $w v$-Lindelöf generalized topological space. (C) 2017 All rights reserved.
\end{abstract}

Keywords: $\quad v$-Lindelöf, $w v$-Lindelöf, G-semiregular generalized topological space.

2010 MSC: 54A05, 54B05, 54C05, 54C10, 54D15, 54D20.

\section{Introduction}

A lot of attention has been made to study properties of covering in topological spaces, which include open and different kind of generalized open sets. Further, several authors have been introduced the generalization of Lindelöf space separately for many reasons and according to the sets that they are interested in such as $[17,22]$. Moreover, in a few last years the generalization of Lindelöf spaces have been extended and generalized to bitopological setting as in [23, 25].

In 1997, essential kind of sets was introduced by Császár [8], namely generalized open sets, that produced generalized topological spaces. Afterwords, a lot of authors have been achieved to generalize the topological notions to generalized topological surroundings. In literature, there are several generalizations of the notion of regular sets, and these are studied separately for different reasons and purposes. In 2008, Császár [13] defined v-regular open (resp. v-regular closed) sets. In 2012, Sarsak [27] introduced and studied $v$-compact (resp. v-Lindelöf) sets in generalized topological spaces. After that in 2014, Arar [5] gave the corresponding definitions of paracompact spaces in generalized topological spaces. In 2015, Kiliçman and Abuage studied some spaces generated by v-regular sets [16]. Also, in [3] and [1] Abuage and Kiliçman introduced nearly $v$-Lindelöf (briefly. $n v$-Lindelöf) and almost $v$-Lindelöf (briefly

\footnotetext{
*Corresponding author

Email addresses: slaa.salem@yahoo.com (Mariam Abuage), akilic@upm.edu.my (A. Kiliçman)
} 
av-Lindelöf) space in generalized topological spaces respectively. Currently, our purpose is to define a new generalization of $v$-Lindelöf space namely; $w v$-Lindelöf.

In the third section, we shall introduce the concept of $w v$-Lindelöf generalized topological spaces, and obtain some results. Furthermore, the relation among $w v$-Lindelöf, $v$-Lindelöf, $n v$-Lindelöf and av-Lindelöf GTS have been given.

In the forth section, some characterizations of the concept of $w v$-Lindelöf subspaces and subsets are investigated. The primary result is that the $w v$-Lindelöf generalized topological space is not a hereditary property. In the fifth section, we shall introduce the effect of some mappings and decompositions. The main result of our study is that almost $(\nu, \mu)$-continuous image of $w v$-Lindelöf generalized topological space is $w v$-Lindelöf.

\section{Preliminaries}

Suppose a nonempty set $X_{G}, P\left(X_{G}\right)$ denotes the power set of $X_{G}$ and $v$ be a nonempty family of $P\left(X_{G}\right)$. The symbol $v$ implies a generalized topology (briefly GT) on $X_{G}$ [9] if the empty set $\emptyset \in v$ and $U_{\gamma} \in v$ where $\gamma \in \Omega$ implies $\bigcup_{\gamma \in \Omega} U_{\gamma} \in v$. The pair $\left(X_{G}, v\right)$ is called generalized topological space (briefly GTS) and we always denote it by GTS $\left(X_{G}, v\right)$ or $X_{G}$. Each element of GT $v$ is said to be $v$-open set and the complement of $v$-open set is called $v$-closed set. Let $A$ be a subset of a GTS $\left(X_{G}, v\right)$, then $i_{v}(A)$ (resp. $\left.c_{v}(A)\right)$ denotes the union of all $v$-open sets contained in $A$ (resp. denotes the intersection of all $v$-closed sets containing in $A$ ), and $X_{G} \backslash A$ denotes the complement of $A, c_{v}\left(X_{G} \backslash A\right)=X_{G} \backslash\left(i_{v} A\right)$. Moreover, $A$ is said to be $v$-regular open (resp. $v$-regular closed) if and only if $A=i_{v} c_{v}(A)$ (resp. $A=c_{v} i_{v}(A)$ ) [13].

If a set $X_{G} \in v$, then a GTS $\left(X_{G}, v\right)$ is called $v$-space [21], and will be denoted by a $v$-space $\left(X_{G}, v\right)$ or a $v$-space $X_{G} . X_{G}$ is said to be quasi-topological space [12], if the finite intersection of $v$-open sets of $v$ belongs to $v$ and denoted by QTS $\left(X_{G}, v\right)$. If $B \subseteq P\left(X_{G}\right)$ and $\emptyset \in B$. Then $B$ is called a $v$-base [10] for $v$ if $\left\{\cup B^{\prime}: B^{\prime} \subseteq B\right\}=v$, and we say that $v$ is generated by $B$. A GT $v$ generated by $v$-regular open sets of a GTS $\left(X_{G}, v\right)$ is said to be $v$-semiregularization [16] of $\left(X_{G}, v\right)$, denoted by GTS $\left(X_{G}, v_{\delta}\right)$. A GTS $X_{G}$ is said to be G-regular [19] if for each $t \in \Lambda_{v}$ and each $v$-closed set $F$ with $t \notin F$, there are disjoint $v$-open sets $U$ and $V$ such that $t \in U$ and $F \cap \Lambda_{v} \subseteq V$, where $\Lambda_{v}$ is the union of all $v$-open sets in $X_{G}$. A GTS $\left(X_{G}, v\right)$ is called submaximal [14] if every $v$-dense set of $X_{G}$ is $v$-open, and is said to be $v$-extremally disconnected [10] if the $v$-closure of every $v$-open set is $v$-open. Moreover, a subset $A$ of a GTS $\left(X_{G}, v\right)$ is called $v$-clopen if it is both $v$-open and $v$-closed subset.

Proposition 2.1 ([16]).

(a) A GTS $\left(\mathrm{X}_{\mathrm{G}}, v\right)$ is $\mathrm{G}$-semiregular if for each point $\mathrm{t} \in \Lambda_{v}$ and each $v$-open set $\mathrm{U}$ containing $\mathrm{t}$, there exists $v$-open set $\mathrm{V}$ such that $\mathrm{t} \in \mathrm{V} \subseteq \mathrm{i}_{v} \mathrm{c}_{v}(\mathrm{~V}) \cap \Lambda_{v} \subseteq \mathrm{U}$.

(b) A GTS $\left(\mathrm{X}_{\mathrm{G}}, v\right)$ is almost $\mathrm{G}$-regular if each point $\mathrm{t} \in \Lambda_{v}$ and each $v$-regular open set $\mathrm{U}$ containing $\mathrm{t}$, there exists $v$-open set $\mathrm{V}$ such that $\mathrm{t} \in \mathrm{V} \subseteq \mathrm{c}_{v} \mathrm{~V} \cap \Lambda_{v} \subseteq \mathrm{U}$.

Definition 2.2. A GTS $\left(X_{G}, v\right)$ is said to be

(a) $v$-Lindelöf [27] if for each $v$-open cover $\left\{U_{\gamma}: \gamma \in \Omega\right\}$ of $\Lambda_{\gamma}$ admits a countable sub-collection $\left\{\mathrm{U}_{\gamma_{\mathrm{n}}}: n \in \mathbb{N}\right\}$ such that $\Lambda_{v}=\bigcup_{n \in \mathbb{N}} \mathrm{U}_{\gamma_{n}}$.

(b) $n v$-Lindelöf [3] (resp. av-Lindelöf [1]) if for each $v$-open cover $\left\{U_{\gamma}: \gamma \in \Omega\right\}$ of $\Lambda_{v}$ admits a countable sub-collection $\left\{\mathrm{U}_{\gamma_{\mathrm{n}}}: \mathrm{n} \in \mathbb{N}\right\}$ such that

$$
\Lambda_{v}=\bigcup_{n \in \mathbb{N}}\left(i_{v} c_{v}\left(u_{\gamma_{n}}\right)\right) \quad\left(\text { resp. } \Lambda_{v}=\bigcup_{n \in \mathbb{N}}\left(c_{v}\left(U_{\gamma_{n}}\right)\right)\right)
$$

Definition $2.3([2])$. A GTS $\left(X_{G}, v\right)$ is called $n v$-paracompact if each $v$-regular open cover of $\Lambda_{v}$ admits a locally finite $v$-open refinement. 
Lemma 2.4 ([13]).

(a) If $\mathrm{F}$ is $v$-closed set then $i_{v}(F)$ is v-regular open.

(b) If $\mathrm{U}$ is v-open set then $\mathrm{c}_{v}(\mathrm{U})$ is $v$-regular closed.

\section{3. $w v$-Lindelöf generalized topological spaces}

Definition 3.1. A GTS $\left(X_{G}, v\right)$ is said to be $w v$-Lindelöf if each $v$-open cover $\left\{U_{\gamma}: \gamma \in \Omega\right\}$ of $\Lambda_{\gamma}$ admits a countable sub-collection $\left\{\mathrm{U}_{\gamma_{n}}: n \in \mathbb{N}\right\}$ such that

$$
\Lambda_{v}=c_{v}\left(\bigcup_{n \in \mathbb{N}} u_{\gamma_{n}}\right)
$$

Proposition 3.2. A GTS $\left(X_{G}, v\right)$ is $w v$-Lindelöf if and only if every collection $\left\{F_{\gamma}: \gamma \in \Omega\right\}$ of $v$-closed sets of $X_{G}$ such that $\left(\bigcap_{\gamma \in \Omega} F_{\gamma}\right) \cap \Lambda_{v}=\emptyset$ admits a countable sub-collection $\left\{\mathrm{F}_{\gamma_{n}}: n \in \mathbb{N}\right\}$ such that $i_{v}\left(\bigcap_{n \in \mathbb{N}} F_{\gamma_{n}}\right) \cap \Lambda_{v}=$ $\emptyset$.

Proof. $(\Rightarrow)$ Let $\left\{F_{\gamma}: \gamma \in \Omega\right\}$ be a collection of $\gamma$-closed sets of $X_{G}$ such that $\left(\bigcap_{\gamma \in \Omega} F_{\gamma}\right) \cap \Lambda_{\gamma}=\emptyset$. Then $\Lambda_{\gamma}=X_{G} \backslash\left(\bigcap_{\gamma \in \Omega} F_{\gamma}\right)=\bigcup_{\gamma \in \Omega}\left(X_{G} \backslash F_{\gamma}\right)$, i.e., the collection $\left\{X_{G} \backslash F_{\gamma}: \gamma \in \Omega\right\}$ is a $v$-open cover of $\Lambda_{\gamma}$. Since $X_{G}$ is $w v$-Lindelöf, there is a countable sub-collection $\left\{X_{G} \backslash F_{\gamma_{n}}: n \in \mathbb{N}\right\}$ such that

$$
\Lambda_{v}=c_{v}\left(\bigcup_{n \in \mathbb{N}}\left(X_{G} \backslash F_{\gamma_{n}}\right)\right)
$$

Thus,

$$
X_{G} \backslash \Lambda_{v}=X_{G} \backslash\left(c_{v}\left(\bigcup_{n \in \mathbb{N}}\left(X_{G} \backslash F_{\gamma_{n}}\right)\right)\right)=i_{v}\left(X_{G} \backslash \bigcup_{n \in \mathbb{N}}\left(X_{G} \backslash F_{\gamma_{n}}\right)\right)=i_{v}\left(\bigcap_{n \in \mathbb{N}} F_{\gamma_{n}}\right) .
$$

So, $i_{v}\left(\bigcap_{n \in \mathbb{N}} F_{\gamma_{n}}\right) \cap \Lambda_{v}=\emptyset$.

$(\Leftarrow)$ Suppose $\left\{U_{\gamma}: \gamma \in \Omega\right\}$ is a $v$-open cover of $\Lambda_{\gamma}$, then $\Lambda_{\gamma}=U_{\gamma \in \Omega} U_{\gamma}$ and $\left\{X_{G} \backslash U_{\gamma}: \gamma \in \Omega\right\}$ is a collection of $v$-closed sets of $X_{G}$. Thus $\left(X_{G} \backslash \bigcup_{\gamma \in \Omega} U_{\gamma}\right) \cap \Lambda_{\gamma}=\emptyset$, i.e., $\bigcap_{\gamma \in \Omega}\left(X_{G} \backslash U_{\gamma}\right) \cap \Lambda_{\gamma}=\emptyset$. By hypothesis, there is a countable sub-collection $\left\{X_{G} \backslash U_{\gamma_{n}}: n \in \mathbb{N}\right\}$ such that $i_{v}\left(\bigcap_{n \in \mathbb{N}}\left(X_{G} \backslash U_{\gamma_{n}}\right) \cap \cap \Lambda_{v}=\emptyset\right.$. Then,

$$
\Lambda_{v}=X_{G} \backslash\left(i_{v}\left(\bigcap_{n \in \mathbb{N}}\left(X_{G} \backslash U_{\gamma_{n}}\right)\right)\right)=c_{v}\left(X_{G} \backslash \bigcap_{n \in \mathbb{N}}\left(X_{G} \backslash U_{\gamma_{n}}\right)\right)=c_{v}\left(\bigcup_{n \in \mathbb{N}} U_{\gamma_{n}}\right),
$$

which implies that a GTS $\left(\mathrm{X}_{\mathrm{G}}, v\right)$ is a $w v$-Lindelöf.

Proposition 3.3. A GTS $\left(\mathrm{X}_{\mathrm{G}}, v\right)$ is $w v$-Lindelöf if and only if every collection $\left\{\mathrm{F}_{\gamma}: \gamma \in \Omega\right\}$ of $v$-closed sets of $\Lambda_{v}$ for which every countable sub-collection $\left\{F_{\gamma_{n}}: n \in \mathbb{N}\right\}$ satisfies $i_{v}\left(\bigcap_{n \in \mathbb{N}} F_{\gamma_{n}}\right) \cap \Lambda_{v} \neq \emptyset$, the intersection $\left(\bigcap_{\gamma \in \Omega} F_{\gamma}\right) \cap \Lambda_{v} \neq \emptyset$.

Proof. $(\Rightarrow)$ Let $\left\{F_{\gamma}: \gamma \in \Omega\right\}$ be a collection of $\gamma$-closed sets of $X_{G}$ for which every countable sub-collection $\left\{F_{\gamma_{n}}: n \in \mathbb{N}\right\}$ satisfies $i_{v}\left(\bigcap_{n \in \mathbb{N}}\left(F_{\gamma_{n}}\right)\right) \cap \Lambda_{\nu} \neq \emptyset$. Assume that $\left(\bigcap_{\gamma \in \Omega} F_{\gamma}\right) \cap \Lambda_{\nu}=\emptyset$, hence

$$
X_{G} \backslash \Lambda_{\gamma}=\bigcap_{\gamma \in \Omega} F_{\gamma} \Rightarrow \Lambda_{v}=X_{G} \backslash \bigcap_{\gamma \in \Omega} F_{\gamma}=\bigcup_{\gamma \in \Omega}\left(X_{G} \backslash F_{\gamma}\right) .
$$

So, $\left\{X_{G} \backslash F_{\gamma}: \gamma \in \Omega\right\}$ forms a $v$-open cover of $\Lambda_{\gamma}$. Since $X_{G}$ is $w v$-Lindelöf, there is a countable subcollection $\left\{X_{G} \backslash F_{\gamma_{n}}: n \in \mathbb{N}\right\}$ such that $\Lambda_{v}=c_{v}\left(\bigcup_{n \in \mathbb{N}}\left(X_{G} \backslash F_{\gamma_{n}}\right)\right)$. Thus,

$$
X_{G} \backslash \Lambda_{v}=X_{G} \backslash c_{v}\left(\bigcup_{n \in \mathbb{N}}\left(X_{G} \backslash F_{\gamma_{n}}\right)\right)=i_{v}\left(X_{G} \backslash \bigcup_{n \in \mathbb{N}}\left(X_{G} \backslash F_{\gamma_{n}}\right)\right)=i_{v}\left(\bigcap_{n \in \mathbb{N}} F_{\gamma_{n}}\right) .
$$

So, $i_{v}\left(\bigcap_{n \in \mathbb{N}} F_{\gamma_{n}}\right) \cap \Lambda_{v}=\emptyset$ which is contradiction. 
$(\Leftarrow)$ Let that $X_{\mathrm{G}}$ is not $w v$-Lindelöf GTS, then there exists $v$-open cover $\left\{\mathrm{U}_{\gamma}: \gamma \in \Omega\right\}$ of $\Lambda_{\gamma}$ with no countable sub-collection $\left\{U_{\gamma_{n}}: n \in \mathbb{N}\right\}$ such that $\Lambda_{v}=c_{\gamma}\left(\bigcup_{n \in \mathbb{N}} U_{\gamma_{n}}\right)$. Then $\Lambda_{v} \neq c_{v}\left(\bigcup_{n \in \mathbb{N}} U_{\gamma_{n}}\right)$ for any countable sub-collection $\left\{U_{\gamma_{n}}: n \in \mathbb{N}\right\}$. It follows that $X_{G} \backslash c_{v}\left(\bigcup_{n \in \mathbb{N}} U_{\gamma_{n}}\right) \cap \Lambda_{v} \neq \emptyset$, i.e., $i_{v}\left(X_{G} \backslash \bigcup_{n \in \mathbb{N}} U_{\gamma_{n}}\right) \cap \Lambda_{v} \neq \emptyset$ or $i_{v}\left(\bigcap_{n \in \mathbb{N}}\left(X_{G} \backslash U_{\gamma_{n}}\right)\right) \cap \Lambda_{v} \neq \emptyset$. Thus $\left\{X_{G} \backslash U_{\gamma}: \gamma \in \Omega\right\}$ is a collection of $v$-closed sets of $X_{G}$ and satisfies $i_{v}\left(\bigcap_{n \in \mathbb{N}}\left(X_{G} \backslash U_{\gamma_{n}}\right)\right) \cap \Lambda_{v} \neq \emptyset$ for which every countable sub-collection $\left\{X_{G} \backslash U_{\gamma_{n}}: n \in \mathbb{N}\right\}$. By hypothesis, $\left(\bigcap_{\gamma \in \Omega} X_{G} \backslash U_{\gamma}\right) \cap \Lambda_{\gamma} \neq \emptyset$, and thus $X_{G} \backslash\left(\bigcup_{\gamma \in \Omega} U_{\gamma}\right) \cap \Lambda_{\gamma} \neq \emptyset$, i.e., $\Lambda_{\gamma} \neq \bigcup_{\gamma \in \Omega} U_{\gamma}$. This is contradiction with the fact that a collection $\left\{U_{\gamma}: \gamma \in \Omega\right\}$ is a $v$-open cover of a $\Lambda_{v}$. Then $X_{G}$ is $w v$-Lindelöf GTS.

Proposition 3.4. Let $\left(\mathrm{X}_{\mathrm{G}}, \mathrm{v}\right)$ be a GTS. If

(a) $\mathrm{X}_{\mathrm{G}}$ is wv-Lindelöf;

(b) every $v$-regular open cover $\left\{\mathrm{U}_{\gamma}: \gamma \in \Omega\right\}$ of $\Lambda_{\gamma}$ admits a countable subcover $\left\{\mathrm{U}_{\gamma_{n}}: n \in \mathbb{N}\right\}$ with $v$-dense union in $\Lambda_{v}$;

(c) each collection $\left\{F_{\gamma}: \gamma \in \Omega\right\}$ of $v$-regular closed sets of $X_{G}$ such that $\left(\bigcap_{\gamma \in \Omega} F_{\gamma}\right) \cap \Lambda_{\gamma}=\emptyset$ has a countable sub-collection $\left\{F_{\gamma_{n}}: n \in \mathbb{N}\right\}$ such that $i_{v}\left(\bigcap_{n \in \mathbb{N}} F_{\gamma_{n}}\right) \cap \Lambda_{v}=\emptyset$, then the relation: $(a) \Rightarrow(b) \Leftrightarrow$ (c) is true. Further, if a GTS $\mathrm{X}_{\mathrm{G}}$ is $\mathrm{G}$-semiregular, then $(\mathrm{b}) \Rightarrow(\mathrm{a})$.

Proof. $(a) \Rightarrow(b)$ : It is obvious since every $v$-regular open set is $v$-open.

(b) $\Leftrightarrow(c):$ If $\left\{F_{\gamma}: \gamma \in \Omega\right\}$ is a collection of $v$-regular closed sets of $X_{G}$ such that $\left(\bigcap_{\gamma \in \Omega} F_{\gamma}\right) \cap \Lambda_{\gamma}=\emptyset$, thus $X_{G} \backslash \Lambda_{\gamma}=\bigcap_{\gamma \in \Omega} F_{\gamma}$, and hence $\Lambda_{\gamma}=\bigcup_{\gamma \in \Omega}\left(X_{G} \backslash F_{\gamma}\right)$, i.e., the collection $\left\{X_{G} \backslash F_{\gamma}: \gamma \in \Omega\right\}$ is a $v$-regular open cover of $\Lambda_{v}$ by $(b)$, there is a countable sub-collection $\left\{X_{G} \backslash F_{\gamma_{n}}: n \in \mathbb{N}\right\}$ with $v$-dense union in $X_{G}$, i.e., $\Lambda_{v}=c_{v}\left(\bigcup_{n \in \mathbb{N}}\left(X_{G} \backslash F_{\gamma_{n}}\right)\right)$. So, $X_{G} \backslash c_{v}\left(\bigcup_{n \in \mathbb{N}}\left(X_{G} \backslash F_{\gamma_{n}}\right)\right) \cap \Lambda_{v}=\emptyset$, that implies

$$
i_{v}\left(X_{G} \backslash \bigcup_{n \in \mathbb{N}}\left(X_{G} \backslash F_{\gamma_{n}}\right)\right) \cap \Lambda_{v}=\emptyset .
$$

Thus $i_{v}\left(\bigcap_{n \in \mathbb{N}} F_{\gamma_{n}}\right) \cap \Lambda_{v}=\emptyset$.

Conversely, suppose that $\left\{U_{\gamma}: \gamma \in \Omega\right\}$ is a $v$-regular open cover of $\Lambda_{\gamma}$. Then $\Lambda_{\gamma}=\bigcup_{\gamma \in \Omega} U_{\gamma},\left\{X_{G} \backslash U_{\gamma}\right.$ : $\gamma \in \Omega\}$ is a collection of $\nu$-regular closed sets of $X_{G}$, hence $X_{G} \backslash\left(U_{\gamma \in \Omega} U_{\gamma}\right) \cap \Lambda_{\gamma}=\emptyset$, i.e., $\left(\bigcap_{\gamma \in \Omega} X_{G} \backslash U_{\gamma}\right) \cap$ $\Lambda_{v}=\emptyset$. By (c), there is a countable sub-collection $\left\{X_{G} \backslash U_{\gamma_{n}}: n \in \mathbb{N}\right\}$ such that $i_{v}\left(\bigcap_{n \in \mathbb{N}}\left(X_{G} \backslash U_{\gamma_{n}}\right)\right) \cap \Lambda_{v}=$ $\emptyset$. Thus,

$$
\Lambda_{v}=X_{G} \backslash i_{v}\left(\bigcap_{n \in \mathbb{N}}\left(X_{G} \backslash U_{\gamma_{n}}\right)\right)=c_{v}\left(X_{G} \backslash \bigcap_{n \in \mathbb{N}}\left(X_{G} \backslash U_{\gamma_{n}}\right)\right)=c_{v}\left(\bigcup_{n \in \mathbb{N}} u_{\gamma_{n}}\right),
$$

and (b) is proved.

(b) $\Rightarrow(a)$ Let $U=\left\{U_{\gamma}: \gamma \in \Omega\right\}$ be a $v$-open cover of $X_{G}$. By G-semiregularity, for each $t \in X_{G}$, $\mathrm{t} \in \mathrm{V}_{\mathrm{t}} \subseteq \mathrm{U}_{\mathrm{t}}$ for some $\mathrm{U}_{\mathrm{t}} \in \mathrm{U}$, and some $v$-regular open set $\mathrm{V}_{\mathrm{t}}$. Thus by (b) there exist $\left\{\mathrm{t}_{\mathrm{n}}: \mathrm{n} \in \mathbb{N}\right\}$ such that

$$
\Lambda_{v}=\bigcup_{n \in \mathbb{N}}\left(V_{t_{n}}\right) \subseteq \bigcup_{n \in \mathbb{N}}\left(u_{t_{n}}\right) \subseteq c_{v}\left(\bigcup_{n \in \mathbb{N}}\left(u_{t_{n}}\right)\right) .
$$

Thus, $\left\{U_{t_{n}}: n \in \mathbb{N}\right\}$ is a countable sub-collection such that $\Lambda_{v}=c_{v}\left(\bigcup_{n \in \mathbb{N}}\left(U_{t_{n}}\right)\right)$, and then $X_{G}$ is a $w v$-Lindelöf GTS.

Definition $3.5([6])$. A GTS $\left(X_{G}, v\right)$ is called $v$-separable if $X_{G}$ contains a countable $v$-dense subset.

Proposition 3.6. If a $v$-space $\left(\mathrm{X}_{\mathrm{G}}, \mathrm{v}\right)$ is $v$-separable, then it is $w v$-Lindelöf.

Proof. Suppose $\left\{\mathrm{U}_{\gamma}: \gamma \in \Omega\right\}$ is $v$-open cover of $v$-separable $v$-space $\left(\mathrm{X}_{\mathrm{G}}, \boldsymbol{v}\right)$. Then $\mathrm{X}_{\mathrm{G}}$ admits a countable $v$-dense subset $D=\left\{t_{1}, t_{2}, \cdots, t_{n}, \cdots\right\}$. Now, for every $t_{k} \in D$, there is $\gamma_{k} \in \Omega$ with $t_{k} \in U_{\gamma_{k}}$. Thus $X_{G}=c_{v}(D)=c_{v}\left(\bigcup_{k \in \mathbb{N}}\left\{t_{k}\right\}\right)=c_{v}\left(\bigcup_{k \in \mathbb{N}} U_{\gamma_{k}}\right)$. This proves that a $v$-space $\left(X_{G}, v\right)$ is $w v$-Lindelöf. 
Question 3.7. Dose the wv-Lindelöf property imply av-Lindelöf?

Our speculation for Question 3.7, that the answer is no, and we can answer it if the GTS $\left(X_{G}, v\right)$ is weak P-G-space as follows.

Definition 3.8. A GTS $\left(X_{G}, v\right)$ is said to be weak P-G-space if for each countable collection $\left\{U_{n}: n \in \mathbb{N}\right\}$ of $v$-open sets in $X_{G}$, then $c_{\gamma}\left(\bigcup_{n \in \mathbb{N}} U_{\gamma_{n}}\right)=\bigcup_{n \in \mathbb{N}} c_{\gamma}\left(U_{\gamma_{n}}\right)$.

Proposition 3.9. In weak P-G-spaces, av-Lindelöf property is equivalent to $w v$-Lindelöf property.

Proof. the proof follows directly from the Definition above.

Proposition 3.10 ([1]). Every almost G-regular av-Lindelöf GTS is nv-Lindelöf.

On using Propositions 3.9, 3.10, we have the following corollary.

Corollary 3.11. A wv-Lindelöf, almost G-regular and weak P-G-spaces is nv-Lindelöf.

Definition 3.12. A GTS $\left(X_{G}, v\right)$ is said to be $n v$-normal [3] if for each $v$-regular closed sets $F_{1}$ and $F_{2}$ with $\mathrm{F}_{1} \cap \mathrm{F}_{2}=\emptyset$, there are disjoint $v$-open sets $\mathrm{U}, \mathrm{V}$ such that $\mathrm{F}_{1} \cap \Lambda_{v} \subseteq \mathrm{U}, \mathrm{F}_{2} \cap \Lambda_{v} \subseteq \mathrm{V}$.

Proposition 3.13 ([3]). Every nv-Lindelöf almost G-regular GTS is nv-normal.

Corollary 3.14. A wv-Lindelöf, almost G-regular and weak P-G-space is nv-normal.

Proof. The proof is directly deduced from Propositions 3.10, 3.13.

Corollary 3.15 ([1]). Every v-extremally disconnected, av-Lindelöf and G-semiregular GTS is v-Lindelöf.

Corollary 3.16. A wv-Lindelöf, v-extremally disconnected, G-semiregular and weak P-G-spaces is v-Lindelöf.

Lemma 3.17. If $\left\{\mathrm{U}_{\alpha}: \alpha \in \Gamma\right\}$ be a locally finite system of sets in a QTS $\left(X_{\mathrm{G}}, v\right)$, then $c_{v}\left(\bigcup_{\alpha \in \Gamma} \mathrm{U}_{\alpha}\right)=$ $\bigcup_{\alpha \in \Gamma} \mathrm{c}_{v}\left(\mathrm{u}_{\alpha}\right)$.

Proof. Obviously, $\bigcup_{\alpha \in \Gamma} c_{v}\left(U_{\alpha}\right) \subseteq c_{v}\left(\bigcup_{\alpha \in \Gamma} U_{\alpha}\right)$. On the other hand, suppose $t \in c_{v}\left(\bigcup_{\alpha \in \Gamma} U_{\alpha}\right)$. Thus $V_{t} \cap\left(\bigcup_{\alpha \in \Gamma} U_{\alpha}\right) \neq \emptyset$ for every $v$-open set $V_{t}$ containing $t$. Now $\left\{U_{\alpha}: \alpha \in \Gamma\right\}$ is $v$-locally finite, so there is a $v$-open set $V_{t}$ containing $t$ intersects only finitely many of the sets $U_{\alpha}$, say $\left\{U_{\alpha_{k}}: k=1,2, \cdots, n\right\}$, i.e., $V_{t} \cap U_{\alpha_{k}} \neq \emptyset$ for each $k=1,2, \cdots$, n. Since every $v$-open set of $V_{t}$ containing $t$ intersects $\bigcup_{\alpha \in \Gamma} U_{\alpha}$, every $v$-open set of $V_{t}$ containing $t$ must be then intersect $\bigcup_{k=1}^{n} U_{\alpha_{k}}$,i.e., $V_{t} \cap \bigcup_{k=1}^{n} U_{\alpha_{k}} \neq \emptyset$. Hence, $t \in$ $c_{v}\left(\bigcup_{k=1}^{n} U_{\alpha_{k}}\right)=\bigcup_{k=1}^{n}\left(c_{v}\left(U_{\alpha_{k}}\right)\right)$, so that for some $k, t \in c_{v}\left(U_{\alpha_{k}}\right) \subseteq \bigcup_{\alpha \in \Gamma} c_{v}\left(U_{\alpha}\right)$. Thus $c_{v}\left(\bigcup_{\alpha \in \Gamma} U_{\alpha}\right) \subseteq$ $\bigcup_{\alpha \in \Gamma} c_{\gamma}\left(U_{\alpha}\right)$, this completes the proof.

Proposition 3.18. A wv-Lindelöf, $\mathrm{G}$-semiregular and $\mathrm{nv}$-paracompact $\mathrm{QTS}\left(\mathrm{X}_{\mathrm{G}}, \mathrm{v}\right)$ is an av-Lindelöf.

Proof. Suppose $\left\{U_{\gamma}: \gamma \in \Omega\right\}$ is a $v$-regular open cover of $\Lambda_{v}$. Since QTS $X_{G}$ is $n v$-paracompact, then this cover admits a locally finite $v$-open refinement $\left\{V_{\alpha}: \alpha \in \Gamma\right\}$. Since $X_{G}$ is $w v$-Lindelöf, there is a countable sub-collection $\left\{V_{\alpha_{n}}: n \in \mathbb{N}\right\}$ such that $\Lambda_{v}=c_{v}\left(\bigcup_{n \in \mathbb{N}} V_{\alpha_{n}}\right)$. By Lemma 3.17, $c_{v}\left(\bigcup_{n \in \mathbb{N}} V_{\alpha_{n}}\right)=$ $\bigcup_{n \in \mathbb{N}} c_{\gamma}\left(V_{\alpha_{n}}\right)$. Choosing, for each $n \in \mathbb{N}, \gamma_{n} \in \Omega$ such that $V_{\alpha_{n}} \subseteq U_{\gamma_{n}}$, thus

$$
\Lambda_{v}=\bigcup_{n \in \mathbb{N}} c_{v}\left(V_{\alpha_{n}}\right) \subseteq \bigcup_{n \in \mathbb{N}} c_{v}\left(u_{\gamma_{n}}\right)
$$

This proves that a QTS $\left(X_{G}, v\right)$ is an av-Lindelöf.

\section{1. wv-Lindelöf subspaces and subsets}

In [27], Sarsak defined the generalized topological subspace in GTS, since a collection $\{U \cap A: U \in v\}$ is the subspace generalized topology on a subset $A$ of a GTS $\left(X_{G}, v\right)$, and $(A, v(A))$ denotes the generalized topological subspace $(A, v(A))$. 
Definition 3.19. Let a GTS $\left(X_{G}, v\right)$ and $A \subseteq X_{G}$, then $A$ is said to be:

(a) $a v(A)$-Lindelöf if for any $v(A)$-open cover $\left\{U_{\gamma}: \gamma \in \Omega\right\}$ of $A \cap \Lambda_{\gamma}$ admits a countable sub-collection $\left\{\mathrm{U}_{\gamma_{\mathrm{n}}}: \mathrm{n} \in \mathbb{N}\right\}$ such that

$$
A \cap \Lambda_{v}=c_{v(A)}\left(\bigcup_{n \in \mathbb{N}} U_{\gamma_{n}}\right)
$$

(b) a $v$-Lindelöf relative to $X_{G}$ if for each $v$-open cover $\left\{U_{\gamma}: \gamma \in \Omega\right\}$ of $\Lambda_{\gamma}$ where $A \cap \Lambda_{\gamma} \subseteq \bigcup_{\gamma \in \Omega}\left(U_{\gamma}\right)$, there exists a countable sub-collection $\left\{\mathrm{U}_{\gamma_{n}}: n \in \mathbb{N}\right\}$ such that

$$
A \cap \Lambda_{v} \subseteq c_{v}\left(\bigcup_{n \in \mathbb{N}} u_{\gamma_{n}}\right)
$$

Proposition 3.20. Let $\mathrm{A}$ be a subset of a GTS $\left(X_{\mathrm{G}}, v\right)$. Then $\mathrm{A}$ is a wv-Lindelöf relative to $\mathrm{X}_{\mathrm{G}}$ if and only if for each collection $\left\{F_{\gamma}: \gamma \in \Omega\right\}$ of $v$-closed sets of $X_{G}$ such that $\left(\bigcap_{\gamma \in \Omega} F_{\gamma}\right) \cap\left(A \cap \Lambda_{\gamma}\right)=\emptyset$, there is a countable sub-collection $\left\{F_{\gamma_{n}}: n \in \mathbb{N}\right\}$ such that $i_{v}\left(\bigcap_{n \in \mathbb{N}} F_{\gamma_{n}}\right) \cap\left(A \cap \Lambda_{v}\right)=\emptyset$.

Proof. Suppose $\left\{\mathrm{F}_{\gamma}: \gamma \in \Omega\right\}$ is a collection of $\gamma$-closed subsets of a GTS $\mathrm{X}_{\mathrm{G}}$ such that

$$
\left(\bigcap_{\gamma \in \Omega} F_{\gamma}\right) \cap\left(A \cap \Lambda_{\gamma}\right)=\emptyset
$$

Then $\left(A \cap \Lambda_{\gamma}\right) \subseteq X_{G} \backslash\left(\bigcap_{\gamma \in \Omega} F_{\gamma}\right)=\bigcup_{\gamma \in \Omega}\left(X_{G} \backslash F_{\gamma}\right)$, so $\left\{X_{G} \backslash F_{\gamma}: \gamma \in \Omega\right\}$ forms a collection of $v$-open subsets of $X_{G}$ covering $A \cap \Lambda_{v}$. By hypothesis, there is a countable subcollection $\left\{X_{G} \backslash F_{\gamma_{n}}: n \in \mathbb{N}\right\}$ such that $A \cap \Lambda_{v} \subseteq c_{v}\left(\bigcup_{n \in \mathbb{N}}\left(X_{G} \backslash F_{\gamma_{n}}\right)\right)$. Hence

$$
\left(X_{G} \backslash\left(c_{v}\left(\bigcup_{n \in \mathbb{N}}\left(X_{G} \backslash F_{\gamma_{n}}\right)\right)\right)\right) \cap\left(A \cap \Lambda_{v}\right)=\emptyset,
$$

i.e., $i_{v}\left(X_{G} \backslash \bigcup_{n \in \mathbb{N}}\left(X_{G} \backslash F_{\gamma_{n}}\right)\right) \cap\left(A \cap \Lambda_{v}\right)=\emptyset$. Thus $i_{v}\left(\bigcap_{n \in \mathbb{N}} F_{\gamma_{n}}\right) \cap\left(A \cap \Lambda_{v}\right)=\emptyset$.

Conversely, let $\left\{U_{\gamma}: \gamma \in \Omega\right\}$ be a collection of $v$-open subsets in $X_{G}$ such that $A \cap \Lambda_{\gamma} \subseteq U_{\gamma \in \Omega} U_{\gamma}$. Then $\left(X_{G} \backslash \bigcup_{\gamma \in \Omega} U_{\gamma}\right) \cap\left(A \cap \Lambda_{\gamma}\right)=\emptyset$, i.e., $\left(\bigcap_{\gamma \in \Omega}\left(X_{G} \backslash U_{\gamma}\right)\right) \cap\left(A \cap \Lambda_{\gamma}\right)=\emptyset$. Since $\left\{X_{G} \backslash U_{\gamma}: \gamma \in \Omega\right\}$ is a collection of $v$-closed subsets of $X_{G}$, by hypothesis there is a countable sub-collection $\left\{X_{G} \backslash U_{\gamma_{n}}: n \in \mathbb{N}\right\}$ such that $i_{v}\left(\bigcap_{n \in \mathbb{N}}\left(X_{G} \backslash U_{\gamma_{n}}\right)\right) \cap\left(A \cap \Lambda_{v}\right)=\emptyset$. Therefore,

$$
A \cap \Lambda_{v} \subseteq X_{G} \backslash i_{v}\left(\bigcap_{n \in \mathbb{N}}\left(X_{G} \backslash U_{\gamma_{n}}\right)\right)=c_{v}\left(X_{G} \backslash\left(\bigcap_{n \in \mathbb{N}}\left(X_{G} \backslash U_{\gamma_{n}}\right)\right)\right)=c_{v}\left(\bigcup_{n \in \mathbb{N}} u_{\gamma_{n}}\right) .
$$

This completes the proof.

Proposition 3.21. Let $a \mathrm{GTS}\left(\mathrm{X}_{\mathrm{G}}, v\right)$ and $\mathrm{A} \subseteq \mathrm{X}_{\mathrm{G}}$, for the following conditions:

(a) $\mathrm{A}$ is $w \mathrm{v}$-Lindelöf relative to $\mathrm{X}_{\mathrm{G}}$;

(b) every $v$-regular open sets $\left\{\mathrm{U}_{\gamma}: \gamma \in \Omega\right\}$ of $\mathrm{X}_{\mathrm{G}}$ that cover $\mathrm{A} \cap \Lambda_{\gamma}$ admits a countable sub-collection

$$
\left\{\mathrm{U}_{\gamma_{\mathrm{n}}}: \mathrm{n} \in \mathbb{N}\right\},
$$

with $v$-dense union in $A \cap \Lambda_{v}$;

(c) each collection $\left\{F_{\gamma}: \gamma \in \Omega\right\}$ of $v$-regular closed sets of $X_{G}$ such that $\bigcap_{\gamma \in \Omega} F_{\gamma} \cap\left(A \cap \Lambda_{\gamma}\right)=\emptyset$ has a countable sub-collection $\left\{F_{\gamma_{n}}: n \in \mathbb{N}\right\}$ such that $i_{v}\left(\bigcap_{n \in \mathbb{N}} F_{\gamma_{n}}\right) \cap\left(A \cap \Lambda_{v}\right)=\emptyset$, then the relation: $(a) \Rightarrow(b) \Leftrightarrow$ (c) is true. Further, if a GTS $\mathrm{X}_{\mathrm{G}}$ is $\mathrm{G}$-semiregular, then $(\mathrm{b}) \Rightarrow(\mathrm{a})$.

Proof. The proof of this proposition is similar to the proof of Proposition 3.4, so we omitted. 
Proposition 3.22. Suppose $a \mathrm{GTS}\left(\mathrm{X}_{\mathrm{G}}, v\right)$ and $\mathrm{A} \subseteq \mathrm{X}_{\mathrm{G}}$, if $\mathrm{A}$ is a $w v(\mathrm{~A})$-Lindelöf then $\mathrm{A}$ is a $w v$-Lindelöf relative to $\mathrm{X}_{\mathrm{G}}$.

Proof. Suppose $\left\{U_{\gamma}: \gamma \in \Omega\right\}$ is a collection of $\nu$-open sets of $X_{G}$ that cover $A \cap \Lambda_{\gamma}$. Then for each $\gamma$, we can find $v$-open set $V_{\gamma}$ of $A \cap \Lambda_{\gamma}$ with $U_{\gamma} \cap\left(A \cap \Lambda_{\gamma}\right)=V_{\gamma}$. Thus $\left\{V_{\gamma}: \gamma \in \Omega\right\}$ is $v(A)$-open cover. Since $A$ is $w v(A)$-Lindelöf, then there is a countable sub-collection $\left\{V_{\gamma_{n}}: n \in \mathbb{N}\right\}$ such that

$$
A \cap \Lambda_{v}=c_{\gamma(A)}\left(\bigcup_{n \in \mathbb{N}} v_{\gamma_{n}}\right) \subseteq c_{v}\left(\bigcup_{n \in \mathbb{N}} u_{\gamma_{n}}\right)
$$

Therefore, $A$ is a $w v$-Lindelöf relative to $X_{G}$.

Question 3.23. Is the converse of Proposition 3.22 above true?

Our speculation for the question above that the answer is no, and the converse of Proposition 3.22 holds if we restrict a GTS $\left(X_{G}, v\right)$ to be a QTS and $A \subseteq X_{G}$ to be a $v$-open subset. We prove that as follows.

Proposition 3.24. Let $\left(\mathrm{X}_{\mathrm{G}}, \mathrm{v}\right)$ be a QTS and $\mathrm{A}$ be a v-open subset of $\mathrm{X}_{\mathrm{G}}$. Then $\mathrm{A}$ is a $w \boldsymbol{w}(\mathrm{A})$-Lindelöf if and only if it is $w v$-Lindelöf relative to $\mathrm{X}_{\mathrm{G}}$.

Proof. $(\Rightarrow)$ The necessity of proof was shown in Proposition 3.22.

$(\Leftarrow)$ Sufficiency, since $A$ be a $v$-open then $A \cap \Lambda_{\nu}=A$. Now, let $\left\{V_{\gamma}: \gamma \in \Omega\right\}$ be a $v(A)$-open cover of $A$, then for each $\gamma \in \Omega, V_{\gamma}=U_{\gamma} \cap A$ where $U_{\gamma}$ is $v$-open, and $A \subseteq U_{\gamma \in \Omega} U_{\gamma}$. Thus by hypothesis there is a countable sub-collection $\left\{U_{\gamma_{n}}: n \in N\right\}$ of $U_{\gamma}$ such that $A \subseteq c_{\gamma}\left(U_{n \in N} U_{\gamma_{n}}\right)$, then

$$
A \subseteq c_{v}\left(\bigcup_{n \in N} u_{\gamma_{n}}\right) \cap A=c_{v}\left(\bigcup_{n \in N}\left(u_{\gamma_{n}} \cap A\right)\right)=c_{v(A)} \bigcup_{n \in N}\left(v_{\gamma_{n}}\right) \text {. }
$$

It follows that a subset $A$ is $w \mathcal{v}(A)$-Lindelöf.

Note that, in Proposition 3.24 above it shows that in a $v$-open set of a GTS $\left(X_{G}, v\right), w v$-Lindelöf property and $w v$-Lindelöf relative to $X_{\mathrm{G}}$ are equivalent. If we consider $\mathrm{X}_{\mathrm{G}}$ itself is a $w v$-Lindelöf GTS, we conclude the following proposition.

Proposition 3.25. Every $v$-regular closed subset of $w v$-Lindelöf and $\mathrm{G}$-semiregular $\mathrm{QTS}\left(\mathrm{X}_{\mathrm{G}}, \boldsymbol{v}\right)$ is $w v$-Lindelöf relative to $\mathrm{X}_{\mathrm{G}}$.

Proof. Let $A$ be a $v$-regular closed subset of $X_{G}$. If $\left\{U_{\gamma}: \gamma \in \Omega\right\}$ is a cover of $A \cap \Lambda_{\gamma}$ by $v$-regular open subsets of $X_{G}$, then $\Lambda_{\gamma}=\left(\bigcup_{\gamma \in \Omega} U_{\gamma}\right) \cup\left(X_{G} \backslash A \cap \Lambda_{\gamma}\right)$. Hence the collection $\left\{U_{\gamma}: \gamma \in \Omega\right\} \cup\left\{X_{G} \backslash A \cap \Lambda_{\gamma}\right\}$ forms a $v$-regular open cover of $\Lambda_{v}$. Since $X_{G}$ is a $w v$-Lindelöf, by Proposition 3.24 there will be a countable sub-collection $\left\{X_{G} \backslash A \cap \Lambda_{\gamma}, U_{\gamma_{1}}, U_{\gamma_{2}}, \cdots\right\}$ such that

$$
\Lambda_{v}=c_{v}\left(\bigcup_{n \in \mathbb{N}}\left(U_{\gamma_{n}} \cup\left(X_{G} \backslash A \cap \Lambda_{v}\right)\right)\right)=c_{v}\left(\left(\bigcup_{n \in \mathbb{N}} U_{\gamma_{n}}\right) \cup X_{G} \backslash A \cap \Lambda_{v}\right) \text {. }
$$

Then,

$$
\Lambda_{v}=c_{v}\left(\bigcup_{n \in \mathbb{N}} u_{\gamma_{n}}\right) \cup c_{v}\left(X_{G} \backslash A \cap \Lambda_{v}\right)=c_{v}\left(\bigcup_{n \in \mathbb{N}} u_{\gamma_{n}}\right) \cup\left(X_{G} \backslash A \cap \Lambda_{v}\right),
$$

but $A \cap \Lambda_{v}$ and $X_{G} \backslash A \cap \Lambda_{v}$ are disjoint. Hence $A \cap \Lambda_{v} \subseteq c_{v}\left(\bigcup_{n \in \mathbb{N}} U_{\gamma_{n}}\right)$. This proves that $A$ is $w v$ Lindelöf relative to $X_{G}$.

Since every $v$-clopen subset is $v$-regular closed, we have the next corollary.

Corollary 3.26. Every $v$-clopen subset of $w v$-Lindelöf and $\mathrm{G}$-semiregular $\mathrm{QTS}\left(\mathrm{X}_{\mathrm{G}}, \mathrm{v}\right)$ is $w \boldsymbol{v}$-Lindelöf relative to $X_{G}$. 
Question 3.27. Is $v$-closed (v-regular open) subset of $w v$-Lindelöf QTS $X_{\mathrm{G}}$ wv-Lindelöf?

We leave the answer for readers.

So, we can say that in general $w v$-Lindelöf property is not a hereditary property.

Definition 3.28. A GTS $\left(X_{G}, v\right)$ is said to be hereditary $w v$-Lindelöf if every subspace of $X_{G}$ is $w v$ Lindelöf.

Proposition 3.29. Let $\left(\mathrm{X}_{\mathrm{G}}, v\right)$ be a $\mathrm{G}$-semiregular GTS. Then $\mathrm{X}_{\mathrm{G}}$ is hereditary $w v$-Lindelöf $\mathrm{GTS}$ if and only if any $A \in v_{\delta}$ is $w v(A)$-Lindelöf.

Proof. $(\Rightarrow)$ Suppose $X_{\mathrm{G}}$ is a G-semiregular GTS and $v$-open hereditary $w v$-Lindelöf. Since $v_{\delta} \subseteq v$, it is obvious that any $A \in v_{\delta}$ implies $A \in v$ and hence $A$ is $w v(A)$-Lindelöf.

$(\Leftarrow)$ Let $V \subseteq X_{G}$ be a $v$-open subset of GTS $X_{G}$. By Proposition 3.24, it is sufficient to prove that $V$ is $w v$-Lindelöf relative to $X_{G}$. Let $\left\{U_{\gamma}: \gamma \in \Omega\right\}$ be a collection of $v$-open subsets of $X_{G}$ such that $\mathrm{V} \cap \Lambda_{\gamma} \subseteq \bigcup_{\gamma \in \Omega} \mathrm{U}_{\gamma}$. By Lemma 2.4, we have $\left\{i_{\nu} c_{\gamma} \mathrm{U}_{\gamma}: \gamma \in \Omega\right\}$ is a collection of $v$-regular open subsets of $X_{G}$. The set $A \cap \Lambda_{\gamma}=\bigcup_{\gamma \in \Omega} i_{v} c_{\gamma}\left(U_{\gamma}\right) \in v_{\delta}$, since $A$ is $w v(A)$-Lindelöf. Then there is a countable sub-collection $\left\{i_{v} c_{\gamma}\left(U_{\gamma_{n}}\right): n \in \mathbb{N}\right\}$ such that

$$
A \cap \Lambda_{v}=c_{v}\left(\bigcup_{n \in \mathbb{N}} i_{v} c_{v}\left(U_{\gamma_{n}}\right)\right) \subseteq c_{v}\left(\bigcup_{n \in \mathbb{N}} c_{v}\left(u_{\gamma_{n}}\right)\right) \subseteq c_{v}\left(\bigcup_{n \in \mathbb{N}}\left(u_{\gamma_{n}}\right)\right) .
$$

Therefore, $\mathrm{V} \cap \Lambda_{v} \subseteq A \cap \Lambda_{v} \subseteq \mathrm{c}_{v}\left(\bigcup_{n \in \mathbb{N}}\left(\mathrm{U}_{\gamma_{n}}\right)\right)$ and this completes the proof.

\section{Mapping properties}

The notions of continuous functions in generalized topological spaces was introduced by Császár [9] in 2002. Let $v$ and $\mu$ be generalized topologies on $X_{G}$ and $Y_{G}$, respectively. Then a function $g:\left(X_{G}, v\right) \rightarrow$ $\left(Y_{G}, \mu\right)$ from a $v$-space $\left(X_{G}, v\right)$ into a $\mu$-space $\left(Y_{G}, \mu\right)$ is called $(\nu, \mu)$-continuous, if and only if $U \in \mu$ implies that $g^{-1}(U) \in v$.

Definition 4.1. Let $A$ be a subset of $\operatorname{GTS}\left(X_{G}, v\right)$, then $A$ is called $v$-preopen (resp. $v$ - $\beta$-open) [11] if $A \subseteq i_{v} c_{v}(A)$ (resp. $A \subseteq c_{v} i_{v} c_{v}(A)$ ). The complement of $v$-preopen (resp. $v$ - $\beta$-open) is said to be $v$ preclosed (resp. $\nu$ - $\beta$-closed), we denote by $\pi$ the class of all $\nu$-preopen sets in $X_{G}$, by $\beta$ the class of all $\nu$ - $\beta$-open sets in $X_{G}$.

Definition 4.2. A function $g:\left(X_{G}, v\right) \longrightarrow\left(Y_{G}, \mu\right)$ is called:

(1) almost $(\nu, \mu)$ - continuous [18], if for each $t \in X_{G}$ and each $\mu$-open set $U$ containing $g(t)$, there is a $v$-open set $V$ with $t \in V$ such that $g(V) \subseteq i_{\mu} c_{\mu}(U)$;

(2) almost $(\pi, \mu)$ - continuous (resp. almost $(\beta, \mu)$ - continuous) [3] if for each $t \in X_{G}$ and each $\mu$-regular open set $\mathrm{U}$ in $\mathrm{Y}_{\mathrm{G}}$ containing $\mathrm{g}(\mathrm{t})$, there is a $v$-preopen (resp. $v$ - $\beta$-open ) set $\mathrm{V}$ containing $t$ such that $\mathrm{g}(\mathrm{V}) \subseteq \mathrm{U}$.

Remark 4.3. Let $g:\left(X_{G}, v\right) \rightarrow\left(Y_{G}, \mu\right)$ be a function between $G_{T S}$ 's $\left(X_{G}, v\right)$ and $\left(Y_{G}, \mu\right)$. Then we have the following implications but the reverse relations may not be true in general:

$$
\text { almost }(\nu, \mu) \text {-continuous } \Rightarrow \text { almost }(\pi, \mu) \text {-continuous } \Rightarrow \text { almost }(\beta, \mu) \text {-continuous. }
$$

Example 4.4. Let $\mathrm{X}_{\mathrm{G}}=\{\mathrm{a}, \mathrm{b}, \mathrm{c}\}$ and $v=\{\emptyset,\{\mathrm{a}, \mathrm{b}\}\}$ be $a \mathrm{GT}$ on $\mathrm{X}_{\mathrm{G}}$. Then $\pi=v \cup\{\{\mathrm{a}\},\{\mathrm{b}\}\}$. Define a function $\mathrm{g}:\left(X_{\mathrm{G}}, v\right) \rightarrow\left(X_{\mathrm{G}}, v\right)$ as follows: $\mathrm{g}(\mathrm{a})=\mathrm{a}, \mathrm{g}(\mathrm{b})=\mathrm{g}(\mathrm{c})=\mathrm{c}$. Then $\mathrm{g}$ is almost $(\pi, \mu)$-continuous function but not almost $(\nu, \mu)$-continuous.

Example 4.5. Let $X_{G}=\{a, b, c\}$ and

$$
v=\{\emptyset,\{a\},\{b\},\{a, b\}\},
$$

be $a \mathrm{GT}$ on $\mathrm{X}_{\mathrm{G}}$. Then $\pi=v$ and $\beta=v \cup\left\{\{\mathrm{a}, \mathrm{b}\},\{\mathrm{a}, \mathrm{c}\}, \mathrm{X}_{\mathrm{G}}\right\}$. Consider a function $\mathrm{g}:\left(\mathrm{X}_{\mathrm{G}}, v\right) \rightarrow\left(\mathrm{X}_{\mathrm{G}}, v\right)$ defined by $\mathrm{g}(\mathrm{a})=\mathrm{g}(\mathrm{b})=\mathrm{b}, \mathrm{g}(\mathrm{c})=\mathrm{a}$. Then $\mathrm{g}$ is almost $(\beta, \mu)$-continuous function without begin almost $(\pi, \mu)$-continuous. 
Proposition 4.6. Let $\mathrm{g}:\left(\mathrm{X}_{\mathrm{G}}, v\right) \rightarrow\left(\mathrm{Y}_{\mathrm{G}}, \mu\right)$ be an almost $(\nu, \mu)$-continuous surjection from a $v$-space $\left(\mathrm{X}_{\mathrm{G}}, v\right)$ into a $\mu$-space $\left(\mathrm{Y}_{\mathrm{G}}, \mu\right)$, if a $v$-space $\mathrm{X}_{\mathrm{G}}$ is $w v$-Lindelöf then a $\mu$-space $\mathrm{Y}_{\mathrm{G}}$ is so.

Proof. Suppose $\left\{U_{\gamma}: \gamma \in \Omega\right\}$ is a $\mu$-open cover of $Y_{G}$. Then by Lemma $2.4\left\{i_{\mu} c_{\mu}\left(U_{\gamma}\right): \gamma \in \Omega\right\}$ is a $\mu$-regular open cover of $Y_{G}$. Since $g$ is almost $(v, \mu)$-continuous, that means $g^{-1}\left(i_{\mu} c_{\mu}\left(U_{\gamma}\right)\right)$ is a $v$-open in a $v$-space $X_{G}$. Thus $\left\{g^{-1}\left(i_{\mu} c_{\mu}\left(U_{\gamma}\right)\right): \gamma \in \Omega\right\}$ is a $v$-open cover of $w v$-Lindelöf $v$-space $\left(X_{G}, v\right)$, then there is a countable sub-collection $\left\{\mathrm{g}^{-1}\left(\mathfrak{i}_{\mu} \mathrm{c}_{\mu}\left(\mathrm{U}_{\gamma_{\mathrm{n}}}\right)\right): \mathrm{n} \in \mathbb{N}\right\}$ such that

$$
\begin{aligned}
X_{\mathrm{G}} & =c_{v}\left(\bigcup_{n \in \mathbb{N}} g^{-1}\left(i_{\mu} c_{\mu}\left(U_{\gamma_{n}}\right)\right)\right) \subseteq c_{v}\left(\bigcup_{n \in \mathbb{N}} g^{-1}\left(c_{\mu}\left(U_{\gamma_{n}}\right)\right)\right) \\
& =c_{v}\left(g^{-1}\left(\bigcup_{n \in \mathbb{N}}\left(c_{\mu}\left(u_{\gamma_{n}}\right)\right)\right) \subseteq c_{v}\left(g^{-1}\left(c_{\mu}\left(\bigcup_{n \in \mathbb{N}}\left(U_{\gamma_{n}}\right)\right)\right)\right) .\right.
\end{aligned}
$$

Since $c_{\mu}\left(\bigcup_{n \in \mathbb{N}}\left(U_{\gamma_{n}}\right)\right)$ is $\mu$-regular closed in a $\mu$-space $Y_{G}$ and $g$ is an almost $(\nu, \mu)$-continuous, we have $\mathrm{g}^{-1}\left(c_{\mu}\left(\bigcup_{n \in \mathbb{N}}\left(U_{\gamma_{n}}\right)\right)\right)$ is $v$-closed in a $v$-space $X_{G}$. Thus

$$
X_{G}=c_{v}\left(g^{-1}\left(c_{\mu}\left(\bigcup_{n \in \mathbb{N}}\left(u_{\gamma_{n}}\right)\right)\right)\right)=g^{-1}\left(c_{\mu}\left(\bigcup_{n \in \mathbb{N}}\left(u_{\gamma_{n}}\right)\right)\right) .
$$

Since $g$ is surjective, $Y_{G}=g\left(X_{G}\right)=g\left(g^{-1}\left(c_{\mu}\left(\bigcup_{n \in \mathbb{N}}\left(U_{\gamma_{n}}\right)\right)\right)\right)=c_{\mu}\left(\bigcup_{n \in \mathbb{N}}\left(U_{\gamma_{n}}\right)\right)$. Then a $\mu$-space $Y_{G}$ is $w \mu$-Lindelöf.

By the definitions above, it is clear that every $(\nu, \mu)$-continuous function is almost $(\nu, \mu)$-continuous then we conclude the following corollary.

Corollary 4.7. $w v$-Lindelöf property is a generalized topological property.

Since every $v$-space under finite intersection is topological space, so by Proposition 3.18 and Proposition 4.6 above, we have the following corollary.

Corollary 4.8. Let $\mathrm{g}:\left(\mathrm{X}_{\mathrm{G}}, \mathrm{v}\right) \rightarrow\left(\mathrm{Y}_{\mathrm{G}}, \tau\right)$ be an almost $(\nu, \tau)$-continuous surjection from a $v$-space $\left(\mathrm{X}_{\mathrm{G}}, \boldsymbol{v}\right)$ into a space $\left(\mathrm{Y}_{\mathrm{G}}, \tau\right)$, if $\mathrm{X}_{\mathrm{G}}$ is $w v$-Lindelöf and $\mathrm{Y}_{\mathrm{G}}$ is semiregular and paracompact then it is almost Lindelö space.

Obviously, if $X_{G} \in v$ in $\operatorname{GTS}\left(X_{G}, v\right)$ then $c_{v}(\emptyset)=\emptyset$, so the following proposition is proved immediately by [14, Theorem 30].

Proposition 4.9. Let $\left(\mathrm{X}_{\mathrm{G}}, v\right)$ be a submaximal and $v$-extremally disconnected $v$-space. Then a function $\mathrm{g}:\left(\mathrm{X}_{\mathrm{G}}, \nu\right) \rightarrow\left(\mathrm{Y}_{\mathrm{G}}, \mu\right)$ is an almost $(\nu, \mu)$-continuous if and only if it is almost $(\beta, \mu)$-continuous.

Corollary 4.10. Let $\mathrm{g}:\left(\mathrm{X}_{\mathrm{G}}, v\right) \rightarrow\left(\mathrm{Y}_{\mathrm{G}}, \mu\right)$ be an almost $(\beta, \mu)$-continuous surjection. If $\mathrm{X}_{\mathrm{G}}$ is submaximal, $v$-extremally disconnected and $w \nu$-Lindelöf $\nu$-space, then a $\mu$-space $\mathrm{Y}_{\mathrm{G}}$ is $w \mu$-Lindelöf.

Proof. The proof follows directly from Proposition 4.6 and Proposition 4.9.

Lemma 4.11. Let a $\left(\mathrm{X}_{\mathrm{G}}, \mathrm{v}\right)$ be a submaximal QTS then every $v$-preopen set is $\mathrm{v}$-open.

Proof. Assume, a subset $V$ is a $v$-preopen, then by [26, Proposition 3.11] $V=U \cap A$ for some $v$-regular open set $U$ and $v$-dense set $A$ of $X_{G}$. Since $\left(X_{G}, v\right)$ is submaximal QTS, so $A$ is $v$-open set of $X_{G}$ and thus $V$ is $v$-open set of $X_{G}$.

Next proposition is proved directly, by Lemma 4.11, so the proof is omitted.

Proposition 4.12. Let $\left(\mathrm{X}_{\mathrm{G}}, \nu\right)$ be a submaximal QTS then a function $\mathrm{g}:\left(\mathrm{X}_{\mathrm{G}}, v\right) \rightarrow\left(\mathrm{Y}_{\mathrm{G}}, \mu\right)$ is an almost $(\nu, \mu)$ continuous if and only if it is almost $(\pi, \mu)$-continuous.

By Proposition 4.6 and Proposition 4.12 the following corollary is concluded. 
Corollary 4.13. Let $\mathrm{g}:\left(\mathrm{X}_{\mathrm{G}}, \tau\right) \rightarrow\left(\mathrm{Y}_{\mathrm{G}}, \mu\right)$ be an almost $(\pi, \mu)$-continuous surjection. If a space $\mathrm{X}_{\mathrm{G}}$ is submaximal and weakly Lindelöf then a $\mu$-space $\mathrm{Y}_{\mathrm{G}}$ is $w \mu$-Lindelöf.

Definition $4.14([4])$. A function $g:\left(X_{G}, \nu\right) \longrightarrow\left(Y_{G}, \mu\right)$ is said to be:

(a) almost $(\nu, \mu)$-open if $g(V) \subseteq i_{\mu} c_{\mu}(g(V))$ for each $v$-open set $V$ in $X_{G}$;

(b) contra $(\nu, \mu)$-continuous if $g^{-1}(U)$ is $\nu$-closed in $X_{G}$ for every $\mu$-open set $U$ in $Y_{G}$.

In [4], Al-Omari, and Noiri showed that if a function $g$ from a $v$-space $\left(X_{G}, v\right)$ into a $\mu$-space $\left(Y_{G}, \mu\right)$ is an almost $(\nu, \mu)$-open and contra $(\nu, \mu)$-continuous, then $g$ is almost $(\nu, \mu)$-continuous. Moreover, if $g$ is a contra $(\nu, \mu)$-continuous and a $\mu$-space $Y_{G}$ is $\mu$-extremally disconnected, then $g$ is almost $(\nu, \mu)$ continuous. On using Proposition 4.6 above, we conclude the following corollaries.

Corollary 4.15. Let $\mathrm{g}:\left(\mathrm{X}_{\mathrm{G}}, \nu\right) \rightarrow\left(\mathrm{Y}_{\mathrm{G}}, \mu\right)$ be an almost $(\nu, \mu)$-open and contra $(\nu, \mu)$-continuous surjection from a $v$-space $\left(\mathrm{X}_{\mathrm{G}}, \nu\right)$ into a $\mu$-space $\left(\mathrm{Y}_{\mathrm{G}}, \mu\right)$, if a $v$-space $\mathrm{X}_{\mathrm{G}}$ is $w v$-Lindelöf, then a $\mu$-space $\mathrm{Y}_{\mathrm{G}}$ is so.

Corollary 4.16. Let $\mathrm{g}:\left(\mathrm{X}_{\mathrm{G}}, \nu\right) \rightarrow\left(\mathrm{Y}_{\mathrm{G}}, \mu\right)$ be a contra $(\nu, \mu)$-continuous and a $\mu$-space $\mathrm{Y}_{\mathrm{G}}$ is $\mu$-extremally disconnected from a $v$-space $\left(\mathrm{X}_{\mathrm{G}}, \nu\right)$ into a $\mu$-space $\left(\mathrm{Y}_{\mathrm{G}}, \mu\right)$, if a $\nu$-space $\mathrm{X}_{\mathrm{G}}$ is $w \boldsymbol{v}$-Lindelöf then $a \mu$-space $\mathrm{Y}_{\mathrm{G}}$ is so.

Definition 4.17. A function $g:\left(X_{G}, v\right) \rightarrow\left(Y_{G}, \mu\right)$ is called:

(i) $\left(\delta, \delta^{\prime}\right)$-continuous [19] (resp. almost $\left(\delta, \delta^{\prime}\right)$-continuous) if for each $t \in X_{G}$ and each $\mu$-regular open set $\mathrm{U}$ containing $\mathrm{g}(\mathrm{t})$, there is a $v$-regular open set $\mathrm{V}$ containing $\mathrm{t}$ such that $\mathrm{g}(\mathrm{V}) \subseteq \mathrm{U}$ (resp. $\mathrm{g}(\mathrm{V}) \subseteq$ $\left.\mathrm{c}_{\mu}(\mathrm{U})\right)$;

(ii) super $(\nu, \mu)$-continuous [20] if for each $t \in X_{G}$ and each $\mu$-open set $U$ containing $g(t)$, there is a $v$-open set $\mathrm{V}$ containing $\mathrm{t}$ such that $\mathrm{g}\left(i_{v} \mathrm{c}_{v} \mathrm{~V}\right) \subseteq \mathrm{U}$;

(iii) $\theta(\nu, \mu)$-continuous [9] (resp. strongly $\theta(\nu, \mu)$-continuous [20]) if for each $t \in X_{G}$ and each $\mu$-open set $\mathrm{U}$ containing $\mathrm{g}(\mathrm{t})$, there is a $v$-open set $\mathrm{V}$ containing $\mathrm{t}$ such that $\mathrm{g}\left(\mathrm{c}_{v} \mathrm{~V}\right) \subseteq \mathrm{c}_{\mu}(\mathrm{U})\left(\right.$ resp. $\left.g\left(\mathrm{c}_{v} \mathrm{~V}\right) \subseteq \mathrm{U}\right)$.

Remark 4.18. From the definition above we obtain the following implications but the reverse relations, in general are not true (see [18-20]).

strongly $\theta(\nu, \mu)$-continuous $\Rightarrow$ super $(\nu, \mu)$-continuous $\Rightarrow\left(\delta, \delta^{\prime}\right)$-continuous $\Rightarrow$ almost $(\nu, \mu)$-continuous.

On using Remark 4.18 and Proposition 4.6 above we conclude the corollary below.

Corollary 4.19. Let $\mathrm{g}:\left(\mathrm{X}_{\mathrm{G}}, \nu\right) \rightarrow\left(\mathrm{Y}_{\mathrm{G}}, \mu\right)$ be a strongly $\theta(\nu, \mu)$-continuous (resp. super $(\nu, \mu)$-continuous, $\left(\delta, \delta^{\prime}\right)$-continuous) surjection, if a $v$-space $X_{\mathrm{G}}$ is $w v$-Lindelöf space then so is a $\mu$-space $\mathrm{Y}_{\mathrm{G}}$.

Lemma 4.20. Let a function $\mathrm{g}:\left(\mathrm{X}_{\mathrm{G}}, \nu\right) \rightarrow\left(\mathrm{Y}_{\mathrm{G}}, \mu\right)$ be a $\theta(\nu, \mu)$-continuous and a $\mu$-space $\mathrm{Y}_{\mathrm{G}}$ is almost $\mathrm{G}$-regular then $\mathrm{g}$ is almost $(\nu, \mu)$-continuous function.

Proof. Let $t \in X_{G}$ and $V$ be any $\mu$-open set of $Y_{G}$ containing $g(t)$. Since $\left(Y_{G}, \mu\right)$ is almost G-regular, we can claim that there exists a $\mu$-regular open set $\mathrm{U}$ such that $\mathrm{g}(\mathrm{t}) \in \mathrm{U} \subseteq \mathrm{c}_{\mu} \mathrm{U} \subseteq i_{\mu} \mathrm{c}_{\mu} \mathrm{V}$. Since $\mathrm{V}$ is $\mu$-open, then by Lemma $2.4 \mathrm{~F}=c_{\mu} i_{\mu}\left(\mathrm{Y}_{\mathrm{G}} \backslash \mathrm{V}\right)$ is $\mu$-regular closed and $\mathrm{g}(\mathrm{t}) \notin \mathrm{F}$. Moreover, there are $\mu$-open sets $V_{1}^{\prime}$ and $V_{2}^{\prime}$ such that $g(t) \in V_{1}^{\prime}, F \subseteq V_{2}^{\prime}$ and $V_{1}^{\prime} \cap V_{2}^{\prime}=\emptyset$. Thus $c_{\mu 1}^{\prime} \cap V_{2}^{\prime}=\emptyset$ and hence $c_{\mu} V_{1}^{\prime} \subseteq Y_{G} \backslash V_{2}^{\prime} \subseteq Y_{G} \backslash F$. So, $g(t) \in V_{1}^{\prime} \subseteq c_{\mu} V_{1}^{\prime} \subseteq Y_{G} \backslash F$. Again $V_{1}^{\prime} \subseteq i_{\mu} c_{\mu} V_{1}^{\prime} \subseteq Y_{G} \backslash F$. Therefore, if $U=i_{\mu} c_{\mu} V_{1}^{\prime}$ then

$$
\mathrm{V}_{1}^{\prime} \subseteq \mathrm{U} \subseteq \mathrm{c}_{\mu} \mathrm{U} \subseteq \mathrm{c}_{\mu} \mathrm{V}_{1}^{\prime} \subseteq i_{\mu} \mathrm{c}_{\mu} \mathrm{V} .
$$

Hence

$$
\mathrm{g}(\mathrm{t}) \in \mathrm{U} \subseteq \mathrm{c}_{\mu} \mathrm{U} \subseteq i_{\mu} \mathrm{c}_{\mu} \mathrm{V} .
$$

Since $g$ is $\theta(\nu, \mu)$-continuous and $c_{\mu} U$ is $\mu$-closed in $Y_{G}$, there is a $v$-open set $H \in \mu$ containing $t$ such that $\mathrm{g}\left(\mathrm{c}_{\nu} \mathrm{H}\right) \subseteq \mathrm{c}_{\mu} \mathrm{U} \subseteq \mathrm{i}_{\mu} \mathrm{c}_{\mu} \mathrm{V}$, and hence $\mathrm{g}(\mathrm{H}) \subseteq i_{\mu} c_{\mu} \mathrm{V}$. Then $\mathrm{g}$ is almost $(\nu, \mu)$-continuous function. 
Since every v-extremally disconnected GTS is almost G-regular, by Lemma 4.20, we conclude the following results.

Corollary 4.21. Let a function $\mathrm{g}:\left(\mathrm{X}_{\mathrm{G}}, \nu\right) \rightarrow\left(\mathrm{Y}_{\mathrm{G}}, \mu\right)$ be a $\theta(\nu, \mu)$-continuous and a $\mu$-space $\mathrm{Y}_{\mathrm{G}}$ is $v$-extremally disconnected then $\mathrm{g}$ is almost $(\nu, \mu)$-continuous function.

Corollary 4.22. Let $\mathrm{g}:\left(\mathrm{X}_{\mathrm{G}}, \nu\right) \rightarrow\left(\mathrm{Y}_{\mathrm{G}}, \mu\right)$ be a $\theta(\nu, \mu)$-continuous surjection and a $\mu$-space $\mathrm{Y}_{\mathrm{G}}$ is almost $\mathrm{G}$ regular ( $v$-extremally disconnected), if a $\nu$-space $\mathrm{X}_{\mathrm{G}}$ is $w \boldsymbol{v}$-Lindelöf space then so is a $\mu$-space $\mathrm{Y}_{\mathrm{G}}$

Proposition 4.23. Let $\mathrm{g}:\left(\mathrm{X}_{\mathrm{G}}, v\right) \rightarrow\left(\mathrm{Y}_{\mathrm{G}}, \mu\right)$ be an almost $\left(\delta, \delta^{\prime}\right)$-continuous surjection from a $v$-space $\mathrm{X}_{\mathrm{G}}$ into a $\mu$-space $\mathrm{Y}_{\mathrm{G}}$. If $\mathrm{X}_{\mathrm{G}}$ is $\mathrm{n} \boldsymbol{v}$-Lindelöf then $\mathrm{Y}_{\mathrm{G}}$ is $w \mu$-Lindelof.

Proof. Let $\left\{\mathrm{U}_{\gamma}: \gamma \in \Omega\right\}$ be a $\mu$-regular open cover of a $\mu$-space $\mathrm{Y}_{\mathrm{G}}$. Let $\mathrm{t} \in \mathrm{X}_{\mathrm{G}}$ and each $\mathrm{U}_{\gamma_{\mathrm{t}}}$ containing $g(t)$. Since $g$ is an almost $\left(\delta, \delta^{\prime}\right)$-continuous, then there is $v$-regular open set $V_{\gamma_{t}}$ of $X_{G}$ containing $t$ such that $\mathrm{g}\left(\mathrm{V}_{\gamma_{\mathrm{t}}}\right) \subseteq \mathrm{c}_{\mu}\left(\mathrm{U}_{\gamma_{\mathrm{t}}}\right)$. So, $\left\{\mathrm{V}_{\gamma_{\mathrm{t}}}: \gamma \in \Omega\right\}$ is $v$-regular open cover of $n v$-Lindelöf $\nu$-space $X_{\mathrm{G}}$. Thus there exists a countable sub-collection $\left\{V_{\gamma_{t_{n}}}: n \in \mathbb{N}\right\}$ such that $X_{G}=\bigcup_{n \in \mathbb{N}}\left(V_{\gamma_{t_{n}}}\right)$. Thus

$$
Y_{G}=g\left(X_{G}\right)=g\left(\bigcup_{n \in \mathbb{N}}\left(V_{\gamma_{t_{n}}}\right)\right)=\bigcup_{n \in \mathbb{N}} g\left(V_{\gamma_{t_{n}}}\right) \subseteq \bigcup_{n \in \mathbb{N}} c_{\mu}\left(U_{\gamma_{t_{n}}}\right) \subseteq c_{\mu}\left(\bigcup_{n \in \mathbb{N}} u_{\gamma_{t_{n}}}\right)
$$

This implies that a $\mu$-space $Y_{G}$ is $w v$-Lindelöf.

Lemma 4.24. Let a function $\mathrm{g}:\left(\mathrm{X}_{\mathrm{G}}, v\right) \rightarrow\left(\mathrm{Y}_{\mathrm{G}}, \mu\right)$ is $\theta(\nu, \mu)$-continuous, then $\mathrm{g}$ is almost $\left(\delta, \delta^{\prime}\right)$-continuous.

Proof. Let $t \in X_{G}$ and a $\mu$-regular open set $U$ of $Y_{G}$ containing $g(t)$. Since $g$ is $\theta(\nu, \mu)$-continuous, then there exists a $v$-open set $V$ of $X_{G}$ containing t such that $g\left(c_{v} V\right) \subseteq c_{\mu}(U)$. Thus $i_{v} c_{v} V$ is $v$-regular open set of $X_{G}$ containing $t$ such that $g\left(i_{\nu} c_{v} V\right) \subseteq g\left(c_{\nu} V\right) \subseteq c_{\mu}(U)$. This implies that $g$ is almost $\left(\delta, \delta^{\prime}\right)$-continuous function.

The converse is not true, as the following example.

Example 4.25. Let $\mathrm{X}_{\mathrm{G}}=\{\mathrm{a}, \mathrm{b}, \mathrm{c}, \mathrm{d}\}$ and $\mathrm{Y}_{\mathrm{G}}=\{\mathrm{r}, \mathrm{s}, \mathrm{t}\}$, we define the $\mathrm{GT}^{\prime} \mathrm{s}$

$$
v=\left\{\emptyset,\{a, b\},\{b, c\},\{b, d\},\{a, b, c\},\{a, b, d\},\{b, c, d\}, X_{G}\right\},
$$

and

$$
\mu=\left\{\emptyset,\{r\},\{s\},\{r, s\}, Y_{G}\right\},
$$

on $\mathrm{X}_{\mathrm{G}}$ and $\mathrm{Y}_{\mathrm{G}}$, respectively. If a function $\mathrm{g}:\left(\mathrm{X}_{\mathrm{G}}, v\right) \rightarrow\left(\mathrm{Y}_{\mathrm{G}}, \mu\right)$ defined as $\mathrm{g}(\mathrm{A})=\mathrm{r}, \mathrm{g}(\mathrm{b})=\mathrm{s}, \mathrm{g}(\mathrm{c})=\mathrm{g}(\mathrm{d})=\mathrm{t}$, then $\mathrm{g}$ is almost $\left(\delta, \delta^{\prime}\right)$-continuous function but it is not $\theta(\nu, \mu)$-continuous, since for a $\mu$-open set $\{\mathrm{r}\}$ containing $\mathrm{g}(\mathrm{a})$ there is no v-open set $\mathrm{V}$ containing a such that $\mathrm{g}\left(\mathrm{c}_{\nu} \mathrm{V}\right) \subseteq \mathrm{c}_{\mu}(\{\mathrm{r}\})$.

Corollary 4.26. Let $\mathrm{g}:\left(\mathrm{X}_{\mathrm{G}}, \nu\right) \rightarrow\left(\mathrm{Y}_{\mathrm{G}}, \mu\right)$ be a $\theta(\nu, \mu)$-continuous surjection from a $\nu$-space $\mathrm{X}_{\mathrm{G}}$ into a $\mu$-space $\mathrm{Y}_{\mathrm{G}}$. If $\mathrm{X}_{\mathrm{G}}$ is $\mathrm{n} v$-Lindelof then $\mathrm{Y}_{\mathrm{G}}$ is $w \mu$-Lindelof.

On using Corollaries 3.11 and 3.14, Proposition 4.6, we have the following result.

Corollary 4.27. Let $\mathrm{g}:\left(\mathrm{X}_{\mathrm{G}}, v\right) \rightarrow\left(\mathrm{Y}_{\mathrm{G}}, \mu\right)$ be an almost $(\nu, \mu)$-continuous surjection from a $v$-space $\left(\mathrm{X}_{\mathrm{G}}, v\right)$ into almost $\mathrm{G}$-regular weak $\mathrm{P}-\mathrm{G}$-space $\left(\mathrm{Y}_{\mathrm{G}}, \mu\right)$, if a $\nu$-space $\mathrm{X}_{\mathrm{G}}$ is $w \boldsymbol{v}$-Lindelöf then a $\mu$-space $\mathrm{Y}_{\mathrm{G}}$ is $\mathrm{n} \mu$-Lindelöf (resp. nu-normal).

By Proposition 3.18 and Proposition 4.6, we also conclude the following.

Corollary 4.28. Let $\mathrm{g}:\left(\mathrm{X}_{\mathrm{G}}, \mathrm{v}\right) \rightarrow\left(\mathrm{Y}_{\mathrm{G}}, \tau\right)$ be an almost $(\nu, \tau)$-continuous surjection from a $v$-space $\left(\mathrm{X}_{\mathrm{G}}, \boldsymbol{v}\right)$ into semiregular and nearly paracompact space $\left(\mathrm{Y}_{\mathrm{G}}, \tau\right)$, if a $\mathrm{v}$-space $\mathrm{X}_{\mathrm{G}}$ is $w \boldsymbol{v}$-Lindelöf, then $\left(\mathrm{Y}_{\mathrm{G}}, \tau\right)$ is almost Lindelöf space. 


\section{References}

[1] M. Abuage, A. Kiliçman, Some Properties and Decomposition on av-Lindelöf generalized topological spaces, (submitted). $1,2.2,3.10,3.15$

[2] M. Abuage, A. Kiliçman, M. S. Sarsak, Generalization of soft v-compact soft generalized topological spaces, arXiv, 2016 (2016), 10 pages. 2.3

[3] M. Abuage, A. Kiliçman, M. S. Sarsak, nv-Lindelöfness, Malay. J. Math. Sci., 11 (2017), 73-86. 1, 2.2, 3.12, 3.13 , 4.2

[4] A. Al-omari, T. Noiri, A unified theory of contra- $(\nu, \lambda)$-continuous functions in generalized topological spaces, Acta Math. Hungar., 135 (2012), 31-41. 4.14, 4

[5] M. Arar, A note on spaces with a countable v-base, Acta Math. Hungar., 144 (2014), 494-498. 1

[6] J. B. T. Ayawan, J. S. R. Canoy, Axioms of Countability in Generalized Topological Spaces, Int. Math. Forum, 8 (2013), 1523-1530. 3.5

[7] F. Cammaroto, G. Santoro, Some counterexamples and properties on generalizations of Lindelöf spaces, Internat. J. Math. Math. Sci., 19 (1996), 737-746.

[8] Á. Császár, Generalized open sets, Acta Math. Hungar., 75 (1997), 65-87. 1

[9] Á. Császár, Generalized topology, generalized continuity, Acta Math. Hungar., 96 (2002), 351-357. 2, 4, 4.17

[10] Á. Császár, Extremally disconneted genealized topologies, Annales Univ. Sci. Budapest Sectio Math., 47 (2004), 91-96. 2

[11] Á. Császár, Generalized open sets in generalized topologies, Acta Math. Hungar., 106 (2005), 53-66. 4.1

[12] Á. Császár, Further remarks on the formula of $\gamma$-interior, Acta Math. Hungar., 113 (2006), 325-332. 2

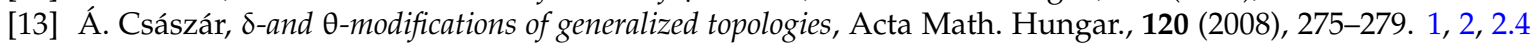

[14] E. Ekici, Generalized submaximal spaces, Acta Math. Hungar., 134 (2011), 132-138. 2, 4

[15] A. J. Fawakhreh, A. Kiliçman, On generalizations of regular-Lindelöf spaces, Internat. J. Math. and Math. Sci., 27 (2001), 535-539.

[16] A. Kiliçman, M. Abuage, On some spaces generated by v-regular sets, JP J. Geom. Topol., 18 (2015), 15-35. 1, 2, 2.1

[17] A. S. Mashhour, M. A. El-Monsef, I. A. Hasanein, T. Noiri, Strongly compact spaces, Delta J. Sci., 8 (1984), 30-46. 1

[18] W. K. Min, Almost continuity on generalized topological spaces, Acta Math. Hungar., 125 (2009), 121-125. 4.2, 4.18

[19] W. K. Min, $\left(\delta, \delta^{\prime}\right)$-continuity on generalized topological spaces, Acta Math. Hungar., 129 (2010), 350-356. 2, 4.17

[20] W. K. Min, Y.-K. Kim, Some strong forms of $\left(\mathrm{g}, \mathrm{g}^{\prime}\right)$-continuity on generalized topological spaces, Honam Math. J., 33 (2011), 85-91. 4.17, 4.18

[21] T. Noriri, Unified characterizations for modifications of $R_{0}$ and $R_{1}$ topological spaces, Rend. Circ. Mat. Palermo, 55 (2006), 29-42. 2

[22] T. Noiri, V. Popa, The unified theory of certain types of generalizations of Lindelöf spaces, Demonstratio Math., 43 (2010), 203-212. 1

[23] Z. Salleh, A. Kiliçman, Pairwise almost Lindelöf bitopological spaces, Malaysian J. of Math. Sci., 1 (2007), 227-238. 1

[24] Z. Salleh, A. Kiliçman, On pairwise nearly Lindelöf bitopological spaces, Far East J. of Math. Sci., 77 (2013), $147-171$.

[25] Z. Salleh, A. Kiliçman, On pairwise weakly Lindelöf bitopological spaces, Bulletin of the Iranian Math. Society, 39 (2013), 469-486. 1

[26] M. S. Sarsak, On some properties of generalized open sets in generalized topological spaces, Demonstratio Math., 46 (2013), 415-427. 4

[27] M. S. Sarsak, On v-compact sets in $\mu$-spaces, Questions Answers in Gen. Topology, 31 (2013), 49-57. 1, 2.2, 3.1 